\title{
Reproducing kernel method for the numerical solution of the 1D Swift-Hohenberg equation
}

\author{
P. Bakhtiari ${ }^{\text {a }}$, S. Abbasbandy ${ }^{a, *}$, R. A. Van Gorder ${ }^{b}$ \\ ${ }^{a}$ Department of Mathematics, Faculty of Science, Imam Khomeini International \\ University, Qazvin, 34149-16818, Iran \\ ${ }^{\mathrm{b}}$ Mathematical Institute, University of Oxford, Andrew Wiles Building, Radcliffe \\ Observatory Quarter, Woodstock Road, Oxford, OX2 6GG, United Kingdom
}

\begin{abstract}
The Swift-Hohenberg equation is a nonlinear partial differential equation of fourth order that models the formation and evolution of patterns in a wide range of physical systems. We study the 1D Swift-Hohenberg equation in order to demonstrate the utility of the reproducing kernel method. The solution is represented in the form of a series in the reproducing kernel space, and truncating this series representation we obtain the $n$-term approximate solution. In the first approach, we aim to explain how to construct a reproducing kernel method without using GramSchmidt orthogonalization, as orthogonalization is computationally expensive. This approach will therefore be most practical for obtaining numerical solutions. GramSchmidt orthogonalization is later applied in the second approach, despite the increased computational time, as this approach will prove theoretically useful when we perform a formal convergence analysis of the reproducing kernel method for the Swift-Hohenberg equation. We demonstrate the applicability of the method through through various test problems for a variety of initial data and parameter values.
\end{abstract}

Key words: Swift-Hohenberg equation; reproducing kernel method; boundary value problem; convergence analysis

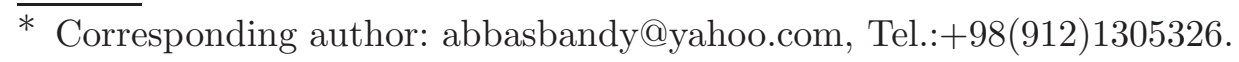




\section{Introduction}

The Swift-Hohenberg equation is a nonlinear partial differential equation of fourth order which has been widely used as a model for the study of pattern formation. It was first put forward by Swift and Hohenberg [25] as a simple model for the Rayleigh-Bernard instability of roll waves. Since then, the Swift-Hohenberg equation has proved an effective model equation for a variety of phenomena in physics and mechanics. Setails of the physics of the Swift-Hohenberg equation can be found in $[8,9,24,26]$. The Swift-Hohenberg equation is defined as [2]

$$
\frac{\partial u}{\partial t}=\lambda u-\left(1+\frac{\partial^{2}}{\partial x^{2}}\right)^{2} u-u^{3},
$$

where $\lambda \in \mathbb{R}$ is a parameter. The Swift-Hohenberg equation is a model equation for a large class of higher-order parabolic model equations. It has a great deal of application, such as the extended Fisher-Kolmogorov equation in statistical mechanics $[2,14,32]$ and a sixth-order equation introduced by Caginalp and Fife [7] in phase field models [15]. Writing equation (1) in a more conventional form, we have

$$
\frac{\partial u}{\partial t}+\frac{\partial^{4} u}{\partial x^{4}}+2 \frac{\partial^{2} u}{\partial x^{2}}+(1-\lambda) u+u^{3}=0
$$

We choose the problem domain $x \in[0,1]$ and $t>0$, along with the boundary and initial conditions

$$
u(0, t)=u(1, t)=0, \quad u_{x x}(0, t)=u_{x x}(1, t)=0, \quad u(x, 0)=f(x) .
$$

Reproducing kernels appeared in the work of Zaremba on boundary value problems involving harmonic and biharmonic functions [31]. Bergman attempted to provide a fundamental framework for the theory of reproducing kernels [5,6], while Aronszajn produced a systematic reproducing kernel space method based on Bergman's works [3,4]. Reproducing kernel methods have proven useful in many areas, including statistics and machine learning, and they play a valuable role in complex analysis, nonlinear system of boundary value problems, nonlinear initial value problems, singular nonlinear two-point periodic boundary value problems and singularly perturbed turning point problems, probability, group representation theory, and the theory of integral operators [1,11-13,16,17,19-21,28,29].

The aim of this paper is to introduce a numerical technique based on reproducing 
kernel Hilbert space methods in order to solve the Swift-Hohenberg initial-boundary value problem (2)-(3). This remainder of this paper is organized as follows. In Section 2, we give a brief introduction to reproducing kernel Hilbert spaces. In Section 3, we present two specific reproducing kernel methods to solve the Swift-Hohenberg initial-boundary value problem. In the first method, we employ non-orthogonal basis functions, while in the second method we using orthogonal basis functions by way of Gram-Schmidt orthogonalization. In Section 4, we provide a convergence analysis of reproducing kernel methods for the Swift-Hohenberg initial-boundary value problem, using the second method. Numerical examples are provided in Section 5 to demonstrate the effectiveness of the proposed method. Concluding remarks, and suggestions for future work, are given in Section 6 .

\section{Reproducing kernel method preliminaries}

To solve the boundary value problem (2)-(3) using the reproducing kernel theory, we need to first discuss some preliminary results.

In order to use the reproducing kernel space to solve (2)-(3), we need to first homogenize the problem. To begin, we define $u(x, t)=\vartheta(x, t)+f(x)$, and consequently we can rewrite (2)-(3) as

$$
\begin{aligned}
& \frac{\partial \vartheta}{\partial t}+\frac{\partial^{4} \vartheta}{\partial x^{4}}+f^{(4)}(x)+2 \frac{\partial^{2} \vartheta}{\partial x^{2}}+2 f^{\prime \prime}(x)+(1-\lambda)(\vartheta(x, t)+f(x))+[\vartheta(x, t)+f(x)]^{3}=0, \\
& \vartheta(0, t)=\vartheta(1, t)=0, \quad \vartheta_{x x}(0, t)=\vartheta_{x x}(1, t)=0, \quad \vartheta(x, 0)=0 .
\end{aligned}
$$

We shall consider the problem domain $\Phi=[0,1] \times[0, \infty)$.

We now present some necessary definitions and theorems in the theory of reproducing kernel spaces. A Hilbert space $\omega$ of functions is called a reproducing kernel Hilbert space if there exists a reproducing kernel $R$ of $\omega$. The existence of the reproducing kernel of a Hilbert space is due to the Riesz Representation Theorem, which states that any continuous linear functional can be represented by an inner product with a unique element of space. Therefore, it is known that the reproducing kernel is unique. Note that the reproducing kernel, $R$, has the following reproducing property

$$
u(\cdot)=\langle u(x), R(x, \cdot)\rangle_{\omega} \quad \text { for all } \quad u \in \omega
$$


Consider the following reproducing kernel space ${ }^{0} \omega_{2}^{2}([0, \infty))$, which is defined as

${ }^{0} \omega_{2}^{2}([0, \infty))=\left\{v(t) \mid v(t), v^{\prime}(t)\right.$ are absolutely continuous functions,

$$
\left.\left.v, v^{\prime}(t), v^{\prime \prime}(t)\right) \in L^{2}([0, \infty)), v(0)=0\right\} .
$$

The name reproducing kernel is motivated by the reproducing property, which is evident in the action of taking the inner product. The inner product and norm for ${ }^{0} \omega_{2}^{2}([0, \infty))$ are given by

$$
\left\langle v_{1}, v_{2}\right\rangle_{0} \omega_{2}^{2}[0, \infty)=\int_{0}^{\infty}\left\{4 v_{1}(t) v_{2}(t)+5 v_{1}^{\prime}(t) v_{2}^{\prime}(t)+v_{1}^{\prime \prime}(t) v_{2}^{\prime \prime}(t)\right\} \mathrm{d} t
$$

and

$$
\|v\|_{\omega_{2}^{2}}=\sqrt{\langle v, v\rangle_{\omega_{2}^{2}}}
$$

respectively. By using the definition of the inner product and the reproducing property (5), we can write the following reproducing kernel [11]:

$$
K_{\eta}(t)= \begin{cases}\frac{1}{12}\left(e^{-2 \eta-2 t}-\frac{1}{12} e^{2 t-2 \eta}-\frac{e^{-\eta-t}}{6}+\frac{e^{t-\eta}}{6}\right), & t \leq \eta, \\ \frac{1}{6}\left(\left(e^{\eta}-e^{-\eta}\right) e^{-t}-\frac{1}{12}\left(e^{4 \eta}-1\right) e^{-2 \eta-2 t}\right), & t>\eta .\end{cases}
$$

Similarly, we define the reproducing kernel space $\omega_{2}^{5}([0,1])$ by

$$
\omega_{2}^{5}([0,1])=\left\{v(x) \mid v^{(4)}(x) \text { is an absolutely continuous, } v^{(5)}(x) \in L^{2}([0,1])\right\} .
$$

The inner product and norm for $\omega_{2}^{5}([0,1])$ are given by

$$
\left\langle v_{1}, v_{2}\right\rangle_{\omega_{2}^{5}}=\sum_{i=0}^{4} v_{1}^{(i)}(0) v_{2}^{(i)}(0)+\int_{0}^{1} v_{1}^{(5)}(x) v_{2}^{(5)}(x) \mathrm{d} x
$$

and

$$
\|v\|_{\omega_{2}^{5}}=\sqrt{\langle v, v\rangle_{\omega_{2}^{5}}}
$$

respectively. By the definition of inner product and according to the the reproducing 
property (5), it follows that its reproducing kernel is [11]:

$$
\begin{aligned}
& \left\{\begin{array}{l}
\frac{1}{12004600204800}(-362880 \zeta(x-1) x(x(x)(x(x(x(91(x-8) x+1588) \\
+664)-8030)-51500)+59380)+59380) 3360 \zeta^{3} x((x)(x(x(x(33(x-9) x \quad x>\zeta,
\end{array}\right. \\
& \left.+91723)-636517)+1905393)+9526965)-22862340) x^{2}+11975040\right) \\
& +210 \zeta^{4} x((x(x(x(x(207(x-9) x-176498)+1270262)-3836868) \\
& +80060040)-152431440) x^{2}+75116160+42 \zeta^{5} x((x(x(x(x)(207(x-9) x \\
& \left.-176498)+1270262)-3836868)+80060040)-152431440) x^{2}+75116160\right) \\
& +28 x\left(11975040+x^{2}(-22862340+x(9526965+x(1905393+x(-636517\right. \\
& +x(91723+33(-9+x) x))))) \zeta^{6}-4 \zeta^{7}(x-1) x(x(x(x(x(x(579(x-8) x \\
& +100987) \text { - 541074) + 1312155) + 10578300) + 87625620) - 210107520+ } \\
& +9 \zeta^{8}(x-1) x(x(x(x(x(x(91(x-8) x+1588)+664)-8030)-51500) \\
& +59380)+59380)+\zeta^{9}\left(-91 x^{9}+819 x^{8}-2316 x^{7}+924 x^{6}+8694 x^{5}\right. \\
& \left.\left.+43470 x^{4}-110880 x^{3}-33022080 x+33081460\right)\right) \text {, } \\
& \frac{1}{12004600204800}\left(x \left(\left(-91 \zeta^{9}+819 \zeta^{8}-2316 \zeta^{7}+924 \zeta^{6}+8694 \zeta^{5}+43470 \zeta^{4}\right.\right.\right. \\
& \left.-110880 \zeta^{3}-33022080 \zeta+33081460\right) x^{8}-3360 \zeta((\zeta(\zeta(\zeta(\zeta(33(\zeta-9) \zeta \\
& \left.+91723)-636517)+1905393)+9526965)-22862340) \zeta^{2}+11975040\right) x^{2} \quad x \leq \zeta . \\
& +28 \zeta((\zeta(\zeta(\zeta(\zeta(33(\zeta-9) \zeta+91723)-636517)+1905393)+9526965) \\
& \left.-22862340) \zeta^{2}+11975040\right) x^{5}+210 \zeta((\zeta(\zeta(\zeta(\zeta(207(\zeta-9) \zeta-176498) \\
& \left.+1270262)-3836868)+80060040)-152431440) \zeta^{2}+75116160\right) x^{3} \\
& +42 \zeta((\zeta(\zeta(\zeta(\zeta(207(\zeta-9) \zeta-176498)+1270262)-3836868) \\
& \left.+80060040)-152431440) \zeta^{2}+75116160\right) x^{4}-362880(\zeta-1) \zeta \\
& \times(\zeta(\zeta(\zeta(\zeta(\zeta(91(\zeta-8) \zeta+1588)+664)-8030)-51500) \\
& +59380)+59380)+9(\zeta-1) \zeta(\zeta(\zeta(\zeta(\zeta(\zeta(91(\zeta-8) \zeta+1588)+664) \\
& -8030)-51500)+59380)+59380) x^{7}-4(\zeta-1) \zeta(\zeta(\zeta(\zeta(\zeta(\zeta(579(\zeta-8) \zeta \\
& \left.+100987)-541074)+1312155)+10578300)+87625620)-210107520) x^{6}\right) \text {, }
\end{aligned}
$$

We next define the binary function space ${ }^{0} \omega_{2}^{(4,2)}(\Phi)$ by

$$
\begin{aligned}
{ }^{0} \omega_{2}^{(5,2)}(\Phi)= & \omega_{2}^{5}([0,1]) \times{ }^{0} \omega_{2}^{2}([0, \infty)) \\
= & \left\{u(x, t) \mid \frac{\partial^{5} u(x, t)}{\partial x^{4} \partial t} \text { is completely continuous in } \Phi, \frac{\partial^{7} u(x, t)}{\partial x^{5} \partial t^{2}} \in L^{2}(\Phi),\right. \\
& \left.u(0, t)=u(1, t)=0, u_{x x}(0, t)=u_{x x}(1, t)=0, u(x, 0)=0\right\} .
\end{aligned}
$$


Before continuing on to the numerical method, we shall need two results.

Theorem 2.1 $[11]^{0} \omega_{2}^{(5,2)}(\Phi)=\omega_{2}^{5}([0,1]) \times{ }^{0} \omega_{2}^{2}([0, \infty))$ is a reproducing kernel space which has reproducing kernel

$$
\Omega_{(\zeta, \eta)}(x, t)=R_{\zeta}(x) \kappa_{\eta}(t)
$$

where $R_{\zeta}(x), \kappa_{\eta}(t)$ are the reproducing kernels of $\omega_{2}^{5}([0,1])$ and ${ }^{0} \omega_{2}^{2}([0, \infty))$, such that for any $u(x, t) \in{ }^{0} \omega_{2}^{(5,2)}(\Phi)$ we have

$$
u(\zeta, \eta)=\left\langle u(x, t), \Omega_{(\zeta, \eta)}(x, t)\right\rangle_{\omega_{2}^{(5,2)}}
$$

It is easy to define the reproducing kernel space $\omega_{2}^{(1,1)}(\Phi)$ such as ${ }^{0} \omega_{2}^{(5,2)}(\Phi)$. Therefore, we can certainly assume that $\Upsilon_{(\zeta, \eta)}(x, y)$ is the reproducing kernel space $\omega_{2}^{(1,1)}(\Phi)$. Moreover, the linear operator $\mathcal{L}:{ }^{0} \omega_{2}^{(5,2)}(\Phi) \longrightarrow{ }^{0} \omega_{2}^{(1,1)}(\Phi)$ is bounded and defined by

$$
(\mathcal{L} \vartheta)(x, t) \equiv \frac{\partial}{\partial t} \vartheta(x, t)+\frac{\partial^{4}}{\partial x^{4}} \vartheta(x, t)+2 \frac{\partial^{2}}{\partial x^{2}} \vartheta(x, t),
$$

where

$$
\mathcal{F}(x, t, \vartheta(x, t)) \equiv(\lambda-1)(\vartheta(x, t)+f(x))-[\vartheta(x, t)+f(x)]^{3}-f^{(4)}(x)-2 f^{\prime \prime}(x)
$$

Hence, Eq. (4) is converted to

$$
\begin{aligned}
& \mathcal{L} \vartheta(x, t)=\mathcal{F}(x, t, \vartheta(x, t)), \\
& \vartheta(0, t)=\vartheta(1, t)=0, \quad \vartheta_{x x}(0, t)=\vartheta_{x x}(1, t)=0, \quad \vartheta(x, 0)=0 .
\end{aligned}
$$

We then set $\varphi_{i}(x, t)=\Upsilon_{\left(x_{i}, t_{i}\right)}(x, t)$, in which $\left\{\left(x_{i}, t_{i}\right)\right\}_{i=1}^{\infty}$ is dense on $\Phi$. Further, we suppose that

$$
\Psi_{i}(x, t)=\mathcal{L}^{*} \varphi_{i}(x, t),
$$

where $\mathcal{L}^{*}$ is the adjoint operator of $\mathcal{L}$.

In the following theorem, the main characteristics of the above concepts are summarized.

Theorem 2.2 [11] If $\left\{\left(x_{i}, t_{i}\right)\right\}_{i=1}^{\infty}$ is dense on $\Phi$, then $\left\{\Psi_{i}(x, t)\right\}_{i=1}^{\infty}$ is a complete system of ${ }^{0} \omega_{2}^{(5,2)} \Phi$. 


\section{Numerical solution method for the Swift-Hohenberg equation}

In this section, we provide two numerical methods for the solution of the SwiftHohenberg equation. The first of these will not employ orthogonalization, while the second will. The first method is therefore less computationally demanding (resulting in faster computation time), while the second method is more useful for convergence analysis (which is considered in the next section). We shall outline both methods here. Since the methods only differ based on the orthogonality of the base functions used in their respective approximations, either method can be used to solve the Swift-Hohenberg equation.

\subsection{Solution approach for Eq. (9) without orthogonalization}

In this section, we propose an iterative algorithm for solving Eq. (9), using the reproducing kernel space. We avoid the use of the Gram-Schmidt orthogonalization process in this method. One benefit to this is that the computational time can be lowered. The solution method of (9) is given in the following theorem.

Theorem 3.1 Let $\left\{\left(x_{i}, t_{i}\right)\right\}_{i=1}^{\infty}$ be a dense set on $\Phi$. If Equation (9) has a unique solution, then then it can be represented as

$$
\vartheta(x, t)=\sum_{i=1}^{\infty} \alpha_{i} \Psi_{i}(x, t)
$$

where the coefficients $\alpha_{i}$ are determined by solving the following semi-infinite system of linear equations $\mathcal{B} \alpha=\mathcal{F}$, in which

$$
\mathcal{B}=\left[\mathcal{L} \Psi_{i}\left(x_{j}, t_{j}\right)\right], \quad i, j=1,2, \ldots, \quad \alpha=\left[\alpha_{1}, \alpha_{2}, \ldots\right]^{T}
$$

and

$$
\mathcal{F}=\left[\mathcal{F}\left(x_{1}, t_{1}, \vartheta\left(x_{1}, t_{1}\right)\right), \mathcal{F}\left(x_{2}, t_{2}, \vartheta\left(x_{2}, t_{2}\right)\right), \ldots\right]^{T}
$$

Proof 3.1 Since $\left\{\left(x_{i}, t_{i}\right)\right\}_{i=1}^{\infty}$ is dense set on $\Phi$, then $\Psi_{i}(x, t)$ is a complete system in ${ }^{0} \omega_{2}^{(5,2)}$, see e.g. [11]. So the analytical solution can be represented as Eq. (11). 
Since

$$
\begin{aligned}
\left\langle\Psi_{j}(x, t), \Psi_{i}(x, t)\right\rangle_{0 \omega_{2}^{(5,2)}} & =\left\langle\mathcal{L}^{*} \varphi_{j}(x, t), \Psi_{i}(x, t)\right\rangle_{0 \omega_{2}^{(5,2)}}=\left\langle\varphi_{j}(x, t), \mathcal{L} \Psi_{i}(x, t)\right\rangle_{0} \omega_{2}^{(1,1)} \\
& =\mathcal{L} \Psi_{i}\left(x_{j}, t_{j}\right)
\end{aligned}
$$

and

$$
\begin{aligned}
\left\langle\vartheta(x, t), \Psi_{j}(x, t)\right\rangle_{0 \omega_{2}^{(5,2)}} & =\left\langle\vartheta(x, t), \mathcal{L}^{*} \varphi_{j}(x, t)\right\rangle_{0 \omega_{2}^{(5,2)}}=\left\langle\mathcal{L} \vartheta(x, t), \varphi_{j}(x, t)\right\rangle_{0 \omega_{2}^{(1,1)}} \\
& =\mathcal{F}\left(x_{j}, t_{j}, \vartheta\left(x_{j}, t_{j}\right)\right) .
\end{aligned}
$$

According to the best approximation principle in Hilbert spaces [22], the coefficients $\alpha_{i}$ are determined by solving the semi-infinite system of linear equations $\mathcal{B} \alpha=\mathcal{F}$, and the proof is complete.

\subsection{Solution approach for Eq. (9) with orthogonalization}

Despite the fact that we can easily obtain solutions as discussed in the previous subsection, we note that by working with orthonormal basis elements, there will be some numerical advantages, particularly for the convergence analysis in the following section. Therefore, we shall derive an orthonormal basis system $\left\{\bar{\Psi}_{i}(x, t)\right\}_{i=1}^{\infty}$ of ${ }^{0} \omega_{2}^{(5,2)}$ from the Gram-Schmidt process applied to $\left\{\Psi_{i}(x, t)\right\}_{i=1}^{\infty}$. To do so, consider basis elements

$$
\bar{\Psi}_{i}(x, t)=\sum_{k=1}^{i} \rho_{i k} \Psi_{k}(x, t),
$$

where $\rho_{i k}$ are orthogonalization coefficients.

Regarding the solution method of Eq. (4), we have the following theorem.

Theorem 3.2 Let $\left\{\left(x_{i}, t_{i}\right)\right\}_{i=1}^{\infty}$ be dense on $\Phi$. If Equation (9) has a unique solution, then the solution satisfies the form

$$
\vartheta(x, t)=\sum_{i=1}^{\infty} \sum_{k=1}^{i} \rho_{i k} \mathcal{F}\left(x_{k}, t_{k}, \vartheta\left(x_{k}, t_{k}\right)\right) \bar{\Psi}_{i}(x, t) .
$$

Proof 3.2 Assume that $\vartheta(x, t)$ is the solution of Eq. (9). By Theorem 2.2, $\vartheta(x, t)$ 
can be expanded in Fourier series as follows:

$$
\begin{aligned}
\vartheta(x, t) & =\sum_{i=1}^{\infty}\left\langle\vartheta(x, t), \bar{\Psi}_{i}(x, t)\right\rangle_{\omega_{2}^{(5,2)}} \bar{\Psi}_{i}(x, t) \\
& =\sum_{i=1}^{\infty} \sum_{k=1}^{i} \rho_{i k}\left\langle\vartheta(x, t), \Psi_{k}(x, t)\right\rangle_{0 \omega_{2}^{(5,2)}} \bar{\Psi}_{i}(x, t) \\
& =\sum_{i=1}^{\infty} \sum_{k=1}^{i} \rho_{i k}\left\langle\vartheta(x, t), \mathcal{L}^{*} \varphi_{k}(x, t)\right\rangle_{0} \omega_{2}^{(5,2)} \bar{\Psi}_{i}(x, t) \\
& =\sum_{i=1}^{\infty} \sum_{k=1}^{i} \rho_{i k}\left\langle\mathcal{L} \vartheta(x, t), \varphi_{k}(x, t)\right\rangle_{0} \omega_{2}^{(1,1)} \bar{\Psi}_{i}(x, t) \\
& =\sum_{i=1}^{\infty} \sum_{k=1}^{i} \rho_{i k} \mathcal{F}\left(x_{k}, t_{k}, \vartheta\left(x_{k}, t_{k}\right)\right) \bar{\Psi}_{i}(x, t)
\end{aligned}
$$

and the proof is complete.

By truncating the series (11), we define the $m$-term approximation to $\vartheta(x, t)$ by

$$
\vartheta_{m}(x, t)=\sum_{i=1}^{m} \kappa_{i} \bar{\Psi}_{i}(x, t)
$$

where

$$
\kappa_{i}=\sum_{k=1}^{i} \rho_{i k} \mathcal{F}\left(x_{k}, t_{k}, \vartheta\left(x_{k}, t_{k}\right)\right) .
$$

Eq. (14) gives the $m$-term approximate solution.

\section{Convergence analysis of the method}

In this section, convergence properties are discussed for the second algorithm. Since this method gives a rather convenient truncated representation for the approximate solution in terms of orthogonal base functions, we choose to work with this second numerical approach. However, we note that one could orthogonalize any base functions used in the approximate solution obtained using the first method, and hence the convergence results we obtain could be obtained for the first method, as well.

Convergence of the sequence $\vartheta_{m}(x, t)$ of approximate solutions is established by the following theorem. 
Theorem 4.1 If $\vartheta(x, t) \in \Phi$, then there exists a constant $C>0$ such that

$$
\begin{aligned}
|\vartheta(x, t)| & \leqslant C\|\vartheta(x, t)\|_{0_{\omega_{2}(5,2)}}, \\
\left|\vartheta_{t}(x, t)\right| & \leqslant C\|\vartheta(x, t)\|_{0_{\omega_{2}(5,2)}}, \\
\left|\vartheta_{x}(x, t)\right| & \leqslant C\|\vartheta(x, t)\|_{\omega_{\omega_{2}^{(5,2)}}}
\end{aligned}
$$

Proof 4.1 For any $(x, t) \in \Phi$, we have

$$
|\vartheta(x, t)|=\left|\left\langle\vartheta(\zeta, \eta), \Upsilon_{(x, t)}(\zeta, \eta)\right\rangle\right|_{\omega_{2}^{(5,2)}} \leqslant\|\vartheta(\zeta, \eta)\|_{\omega_{\omega_{2}^{(5,2)}}}\left\|\Upsilon_{(x, t)}(\zeta, \eta)\right\|_{\omega_{\omega_{2}^{(5,2)}}},
$$

therefore, there exists a constant $C_{1}>0$ such that

$$
|\vartheta(x, t)| \leqslant C_{1}\|\vartheta(\zeta, \eta)\|_{\omega_{2}^{(5,2)}} .
$$

Also, we have

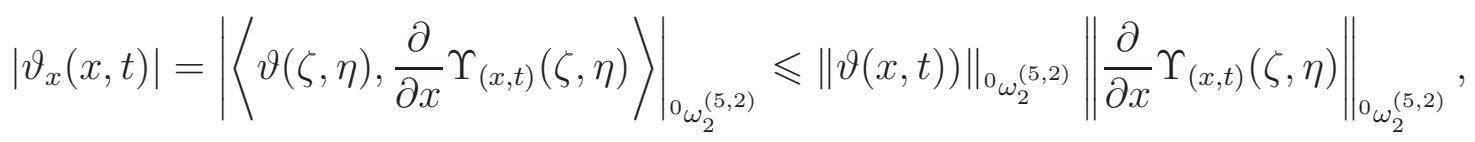

then there exists a constant $C_{2}>0$ such that

$$
\left|\vartheta_{x}(x, t)\right| \leqslant C_{2}\|\vartheta(\zeta, \eta)\|_{\omega_{2}^{(5,2)}} .
$$

In a similar manner, there exist constant $C_{3}>0$ such that $\left|\vartheta_{t}(x, t)\right| \leqslant C_{3}\|\vartheta(x, t)\|_{\omega_{2}^{(5,2)}}$. Let $C=\max \left\{C_{1}, C_{2}, C_{3}\right\}$, then the proof is completed.

As a direct consequence of the above theorem, we have the following result.

Theorem 4.2 If $\vartheta_{m}(x, t) \stackrel{\|\cdot\|_{\omega_{2}^{(5,2)}}}{\longrightarrow} \bar{\vartheta}(x, t)$ as $m \rightarrow \infty,\left(x_{m}, t_{m}\right) \longrightarrow(x, t)$ as $m \rightarrow \infty,\left\|\vartheta_{m}(x, t)\right\|_{0_{2}^{(4,2)}}$ is bounded, and $\mathcal{F}(x, t, \vartheta(x, t))$ is continuous, then

$$
\mathcal{F}\left(x_{m}, t_{m}, \vartheta_{m-1}\left(x_{m}, t_{m}\right)\right) \longrightarrow \mathcal{F}(x, t, \bar{\vartheta}(x, t)), \quad m \rightarrow \infty .
$$

\section{Proof 4.2 Since}

$$
\begin{aligned}
\left|\vartheta_{m-1}\left(x_{m}, t_{m}\right)-\bar{\vartheta}(x, t)\right| & \left.=\mid \vartheta_{m-1}\left(x_{m}, t_{m}\right)-\vartheta_{m-1}(x, t)+\vartheta_{m-1}(x, t)-\bar{\vartheta}(x, t)\right) \mid \\
& \leqslant\left|\partial_{x} \vartheta_{m-1}\right|\left|x_{m}-x\right|+\left|\partial_{t} \vartheta_{m-1}\right|\left|t_{m}-t\right|+\left|\vartheta_{m-1}(x, t)-\bar{\vartheta}(x, t)\right|
\end{aligned}
$$

from the given condition $\vartheta_{m} \stackrel{\|\cdot\|_{\omega_{2}(5,2)}}{\longrightarrow} \bar{\vartheta}$ as $m \rightarrow \infty$, and by the boundedness of $\left\|\vartheta_{m}\right\|_{\omega_{2}^{(5,2)}}$ and Theorem 4.1, for any $(x, t) \in \Phi$, we get

$$
\left|\vartheta_{m-1}\left(x_{m}, t_{m}\right)-\bar{\vartheta}(x, t)\right| \rightarrow 0, \quad m \rightarrow \infty .
$$


The continuation of $\mathcal{F}(x, t, \vartheta(x, t))$ implies that

$$
\mathcal{F}\left(x_{m}, t_{m}, \vartheta_{m-1}\left(x_{m}, t_{m}\right)\right) \longrightarrow \mathcal{F}(x, t, \vartheta(x, t))
$$

as $m \rightarrow \infty$, which completes the proof.

Theorem 4.3 Suppose that $\left\|\vartheta_{m}(x, t)\right\|$ is bounded in (14). If $\left\{\left(x_{i}, t_{i}\right)\right\}_{i=1}^{\infty}$ is dense on $\Phi$, then the $m$-term approximate solution $\vartheta_{m}(x, t)$ derived from the above method converges to the exact solution $\vartheta(x, t)$ of Eq. (9), and

$$
\vartheta(x, t)=\sum_{i=1}^{\infty} \kappa_{i} \bar{\Psi}_{i}(x, t)
$$

where $\kappa_{i}$ is given by Eq. (15).

Proof 4.3 First, we will prove the convergence of $\vartheta_{m}$. By Eq. (14), we infer that

$$
\vartheta_{m+1}(x, t)=\vartheta_{m}(x, t)+\kappa_{m+1} \bar{\Psi}_{m+1}(x, t) .
$$

From the orthogonality of $\left\{\bar{\Psi}_{i}(x, t)\right\}_{i=1}^{\infty}$, we conclude that

$$
\left\|\vartheta_{m+1}\right\|_{0 \omega_{2}^{(5,2)}}=\left\|\vartheta_{m}\right\|_{0 \omega_{2}^{(5,2)}}+\kappa_{m+1}^{2}
$$

Hence it holds that $\left\|\vartheta_{m+1}\right\|_{\omega_{2}^{(5,2)}} \geqslant\left\|\vartheta_{m}\right\|_{0 \omega_{2}^{(5,2)}}$. By the boundedness of $\left\|\vartheta_{m}\right\|_{0 \omega_{2}^{(5,2)}}$, $\left\|\vartheta_{m}\right\|_{\omega_{2}^{(5,2)}}$ is convergent and there exists a constant $c$ such that

$$
\sum_{i=1}^{\infty} \kappa_{i}^{2}=c
$$

This implies that $\kappa_{i} \in l^{2}, \quad i=1,2, \ldots$ If $n>m$, then using the orthogonality of $\vartheta_{m+1}(x, t)-\vartheta_{m}(x, t)$ we have

$$
\begin{aligned}
\left\|\vartheta_{n}(x, t)-\vartheta_{m}(x, t)\right\|_{\omega_{2}^{(5,2)}}^{2} & \\
& =\left\|\vartheta_{n}(x, t)-\vartheta_{n-1}(x, t)+\vartheta_{n-2}(x, t)+\cdots+\vartheta_{m+1}(x, t)-\vartheta_{m}(x, t)\right\|_{{ }_{\omega_{2}(5,2)}}^{2} \\
& =\left\|\vartheta_{n}(x, t)-\vartheta_{n-1}(x, t)\right\|_{\omega_{\omega_{2}^{(5,2)}}}^{2}+\cdots+\left\|\vartheta_{m+1}(x, t)-\vartheta_{m}(x, t)\right\|_{{ }_{\omega_{2}^{(5,2)}}^{2}} \\
& =\sum_{i=m+1}^{n} \kappa_{i}^{2} \longrightarrow 0, \quad m \longrightarrow \infty
\end{aligned}
$$

The completeness of ${ }^{0} \omega_{2}^{(5,2)}$ shows that $\vartheta_{m} \stackrel{\|\cdot\|_{0} \omega_{2}^{(5,2)}}{\longrightarrow} \bar{\vartheta}$ as $m \longrightarrow \infty$. Second, we 
will prove that $\bar{\vartheta}$ is the solution of Eq. (9). To this end, we conclude from (14) that

$$
\bar{\vartheta}(x, t)=\sum_{i=1}^{\infty} \kappa_{i} \bar{\Psi}_{i}(x, t)
$$

Since

$$
(\mathcal{L} \bar{\vartheta})\left(x_{m}, t_{m}\right)=\sum_{i=1}^{\infty} \kappa_{i}\left\langle\mathcal{L} \bar{\Psi}_{i}, \varphi_{m}\right\rangle_{\omega_{\omega}^{(5,2)}}=\sum_{i=1}^{\infty} \kappa_{i}\left\langle\bar{\Psi}_{i}, \Psi_{m}\right\rangle_{\omega_{2}^{(5,2)}},
$$

it follows that

$$
\sum_{j=1}^{m} \rho_{m j}(\mathcal{L} \bar{\vartheta})\left(x_{m}, t_{m}\right)=\sum_{i=1}^{\infty} \kappa_{i}\left\langle\bar{\Psi}_{i}, \sum_{j=1}^{m} \rho_{m j} \Psi_{j}\right\rangle_{0_{\omega_{2}^{(5,2)}}}=\sum_{i=1}^{\infty} \kappa_{i}\left\langle\bar{\Psi}_{i}, \bar{\Psi}_{m}\right\rangle_{\omega_{2}(5,2)}=\kappa_{m} .
$$

If $m=1$, then

$$
(\mathcal{L} \bar{\vartheta})\left(x_{1}, t_{1}\right)=\mathcal{F}\left(x_{1}, t_{1}, \vartheta_{0}\left(x_{1}, t_{1}\right)\right) .
$$

Also, for $m=2$, we have

$$
\rho_{21}(\mathcal{L} \bar{\vartheta})\left(x_{1}, t_{1}\right)+\rho_{22}(\mathcal{L} \bar{\vartheta})\left(x_{2}, t_{2}\right)=\rho_{21} \mathcal{F}\left(x_{1}, t_{1}, \vartheta_{0}\left(x_{1}, t_{1}\right)\right)+\rho_{22} \mathcal{F}\left(x_{2}, t_{2}, \vartheta_{0}\left(x_{2}, t_{2}\right)\right) .
$$

It is clear that

$$
(\mathcal{L} \bar{\vartheta})\left(x_{2}, t_{2}\right)=\mathcal{F}\left(x_{2}, t_{2}, \vartheta_{0}\left(x_{2}, t_{2}\right)\right) .
$$

By induction, we conclude

$$
(\mathcal{L} \bar{\vartheta})\left(x_{j}, t_{j}\right)=\mathcal{F}\left(x_{j}, t_{j}, \vartheta_{j-1}\left(x_{j}, t_{j}\right)\right) .
$$

For any $(\mu, \varrho) \in \Phi$, there exists a subsequence $\left\{\left(x_{m_{j}}, t_{m_{j}}\right)\right\}_{j=1}^{\infty}$ converging to $(\mu, \varrho)$ since $\left\{\left(x_{i}, t_{i}\right)\right\}_{i=1}^{\infty}$ is dense in $\Phi$. Thus, by the convergence of $\vartheta_{m}$ and Theorem 4.2, we conclude that

$$
(\mathcal{L} \bar{\vartheta})(\mu, \varrho)=\mathcal{F}(\mu, \varrho, \bar{\vartheta}(\mu, \varrho)) .
$$

That is, $\bar{\vartheta}(x, t)$ is the solution of Eq. (9) and

$$
\vartheta(x, t)=\sum_{i=1}^{\infty} \kappa_{i} \bar{\Psi}_{i}(x, t) .
$$

The proof is complete.

Theorem 4.4 Assume $\vartheta(x, t)$ is the solution of Eq. (9) and $\tau_{m}(x, t)$ is the approximate error between $\vartheta_{m}(x, t)$ and $\vartheta(x, t)$. Then the error $\tau_{m}(x, t)$ is monotone decreasing in the sense of $\|\cdot\|_{\omega_{2}^{(5,2)}}$. 
Proof 4.4 From (16) and according to the proof of Theorem 4.3, it follows that

$$
\left\|\tau_{m}\right\|_{\omega_{2}^{(5,2)}}^{2}=\left\|\sum_{i=m+1}^{\infty} \kappa_{i} \bar{\Psi}_{i}(x, t)\right\|_{\omega_{\omega_{2}^{(5,2)}}^{2}}^{2}=\sum_{i=m+1}^{\infty} \kappa_{i}^{2} .
$$

Eq. (23) shows that the error $\tau_{m}$ is monotone decreasing in the sense of $\|\cdot\|_{\omega_{2}^{(5,2)}}$.

\section{Numerical experiments}

To verify the applicability and accuracy of the proposed numerical method outlined in Section 3, we choose various initial conditions and parameter values $\lambda$, and perform several numerical experiments. For a sufficient number of iterations, we show that the results converge for both methods, in agreement with the theoretical results of Section 4. In order to minimize computational time, we employ the first method. We find that taking approximate numerical solutions with $n=20$ is sufficient to give solutions with low error, so we choose $n=20$ for the numerical experiments.

Let $E(x, t)$ denote the residual error at the point $(x, t) \in \Phi$. In Tables 1-2, we provide $E(x, t)$ and the values of the approximate solution $u_{n}(x, t)$. We choose the initial condition $u(x, 0)=f(x)$ so that it satisfies the boundary conditions $f(0)=f(1)=0$ and $f^{\prime \prime}(0)=f^{\prime \prime}(1)=0$. We find low values of the residual error for various choices of $\lambda$ and initial conditions, which demonstrates that the numerical approach is rather accurate, and with relatively few terms needed to ensure such a high degree of accuracy.

We may compare our results with those of [26]. In particular, comparing our Table 1 results for $\lambda=-0.7$ with their corresponding results, we find that the minimum residual error of the optimal values of parameters in [26] is $1.16711 \times 10^{-7}$, while our approach gives much smaller residual errors of the order $10^{-14}$ to $10^{-12}$.

The effects of the initial condition and the parameter $\lambda$ on the solution of the

problem (2)-(3) are displayed graphically through Figures 1-6. We give plots of approximate numerical solutions for various initial conditions specific values of $\lambda$, corresponding to the selections used in Tables 1-??. The solutions retain the unimodal properties of the initial conditions for small time, maintaining the overall envelope of the initial conditions. As time increases, the dynamics away from the initial condition will be determined by the value of $\lambda$ selected. 


\section{Conclusions}

In this work, we have proposed two numerical solution approaches for the 1D SwiftHohenberg equation on the basis of the reproducing kernel Hilbert space. For either approach given in Section 3, the solution is represented using a series contained in the reproducing kernel space, and a truncated approximate solution is obtained. The two approaches differ in that the first does not require orthogonalization of the base functions (thereby saving on computational time), while the second approach employs an orthogonalization procedure so that the base functions are mutually orthogonal. Despite the increased computational time, the second approach is theoretically useful, as it allows for a more straightforward convergence analysis. This convergence analysis was given in Section 4. Therefore, there are benefits to both approaches. The results of our numerical experiments demonstrate that the present method is an accurate and reliable numerical technique for the solution of the 1D Swift-Hohenberg equation, and could be extended for use in solving other nonlinear partial differential equations.

One possible extension of our approach would be to consider the solution of 2D or 3D Swift-Hohenberg equations, since it is such higher dimensional equations which permit the formation of interesting patterns [10,18]. Explicit construction of the reproducing kernels in higher dimensional space will be much more complicated, but in principle the numerical approach should be similar $[11,30]$. One approach is to construct the higher dimensional reproducing kernel space as a direct product of lower dimensional or 1D reproducing kernel spaces [4]. Recent applications of reproducing kernel methods in 2D spatial domains have been discussed in [23,27].

\section{References}

[1] S. Abbasbandy, R.A. Van Gorder, P. Bakhtiari, Reproducing kernel method for the numerical solution of the Brinkman-Forchheimer momentum equation, J. Comput. Appl. Math., 311 (2017) 262-271.

[2] F. T. Akyildiz, D. A. Siginer, K. Vajravelu, R. A. Van Gorder, Analytical and numerical results for the Swift-Hohenberg equation, Appl. Math. Comput., 216 (2010) 221-226. 
[3] N. Aronszajn, The theory of reproducing kernels and their applications, Cambridge Philosophy Society Proceedings, 39 (1943) 133-153.

[4] N. Aronszajn, Theory of reproducing kernels, Transactions of the American Mathematical Society, 68 (1950) 337-404.

[5] S. Bergman, Uber Kurvenintegale von Funktionen zweier komplexen Veranderlichen, die Differential gleichungen befriedigen, Math. Z., 32 (1930) 386-406.

[6] S. Bergman, The approximation of functions satisfying a linear partial differential equation, Duke Math. J., 6 (1940) 537-561.

[7] G. Caginalp, P.C. Fife, Higher order phase field models and detailed anisotropy, Phys. Rev. B., 34 (1986) 4940-4943.

[8] J. Chaparova, L.A. Peletier, S. Tersian, Existence and nonexistence of nontrivial solutions of semilinear sixth order ordinary differential equations, Appl. Math. Lett., 17 (2004) 1207-1212.

[9] J. Chaparova, L.A. Peletier, S. Tersian, Existence and nonexistence of nontrivial solutions of semilinear fourth- and sixth-order ordinary differential equations, Adv. Diff. Eqns., 8 (2003) 1237-1258.

[10] M. C. Cross, P. C. Hohenberg, Pattern formation outside of equilibrium, Rev. Mod. Phys., 65 (1993) 851.

[11] M.G. Cui, Y.Z. Lin, Nonlinear Numerical Analysis in Reproducing Kernel Space, Nova Science Pub. Inc., Hauppauge, 2009.

[12] M.G. Cui, F.Z. Geng, Solving singular two-point boundary value problem in reproducing kernel space, J. Comput. Appl. Math., 205 (2007) 6-15.

[13] M.G. Cui, F.Z. Geng, A computational method for solving third-order singularly perturbed boundary-value problems, Appl. Math. Comput., 198 (2008) 896-903.

[14] G.T. Dee, W. van Saarloos, Bistable systems with propagating fronts leading to pattern formation, Phys. Rev. Lett., 60 (1988) 2641-2644.

[15] R.A. Gardner, C.K.R.T. Jones, Traveling waves of a perturbed diffusion equation arising in a phase field model, Indiana Univ. Math. J., 38 (1989) 1197-1222.

[16] F.Z. Geng, M.G. Cui, Solving singular nonlinear two-point boundary value problems in the reproducing kernel space, J. Korean Math. Soc., 45 (2008) 631-644. 
[17] F. Z. Geng, S. P. Qian, S. Li, A numerical method for singularly perturbed turning point problems with an interior layer, J. Comput. Appl. Math., 255 (2014) 97-105.

[18] H. S. Greenside, W. M. Coughran Jr., Nonlinear pattern formation near the onset of Rayleigh-Bénard convection, Phys.l Rev. A, 30 (1984) 398.

[19] X.Y. Li, B.Y. Wu, Error estimation for the reproducing kernel method to solve linear boundary value problems, J. Comput. Appl. Math., 243 (2013) 10-15.

[20] X.Y. Li, B.Y. Wu, A novel method for nonlinear singular fourth order four-point boundary value problems, Comput. Math. Appl., 62 (2011) 27-31.

[21] X.Y. Li, B.Y. Wu, A continuous method for nonlocal functional differential equations with delayed or advanced arguments, J. Math. Anal. Appl., 409 (2014) 485-493.

[22] M. Mohammadi, R. Mokhtari, A reproducing kernel method for solving a class of nonlinear system of PDEs, Math. Model. Anal., 19 (2014) 180-198.

[23] M. Mohammadi, R. Mokhtari, H. Panahipour, A Galerkin-reproducing kernel method: Application to the 2D nonlinear coupled Burgers' equations, Engineering Analysis with Boundary Elements, 37 (2013) 1642-1652.

[24] S. S. Perez-Moreno, S. R. Chavarria, G. R. Chavarria, Numerical Solution of the Swift-Hohenberg Equation, Experimental and Computational Fluid Mechanics, Environmental Science and Engineering, (2013) 409-416.

[25] J.B. Swift, P.C. Hohenberg, Hydrodynamic ffuctuations at the convective instability, Phys. Rev. A 15 (1977) 319-328.

[26] K. Vishal, S. Das, S. H. Ong, P. Ghosh, On the solutions of fractional Swift Hohenberg equation with dispersion, Appl. Math. and Comput., 219 (2013) 5792-5801.

[27] W. Wang, M. Yamamoto, B. Han, Two-dimensional parabolic inverse source problem with final overdetermination in reproducing kernel space, Chinese Annals of Mathematics, Series B, 35 (2014) 469-482.

[28] Y.L. Wang, L. Chao, Using reproducing kernel for solving a class of partial differential equation with variable-coefficients, Appl. Math. Mech. Engl. Ed., 29(1) (2008) 129137.

[29] Y.L. Wang, X.J. Cao, X.N. Li, A new method for solving singular fourth-order boundary value problems with mixed boundary conditions, Appl. Math. Comput., 217 (2011) 7385-7390. 
[30] H. Wendland, Scattered data approximation. Vol. 17. Cambridge University Press (2004).

[31] S. Zaremba, Sur le calcul numerique des founctions demandness dans le problems de dirichlet et le problems hydrodynamique, Bulletin International de I Academie des Sciences de Cracovie, 68 (1908) 125-195.

[32] W. Zimmerman, Propagating fronts near a Lifschitz point, Phys. Rev. Lett., 66 (1991) 1546. 


\begin{tabular}{ccccc}
\hline & $E(x, t)$ & $E(x, t)$ & $E(x, t)$ & $E(x, t)$ \\
$(x, t)$ & for $\lambda=-0.7$ & for $\lambda=0.3$ & for $\lambda=1.0$ & for $\lambda=8.0$ \\
\hline$(0.1,0.1)$ & $3.06577 \times 10^{-13}$ & $4.42522 \times 10^{-13}$ & $3.93843 \times 10^{-13}$ & $2.51455 \times 10^{-14}$ \\
$(0.2,0.2)$ & $6.70665 \times 10^{-14}$ & $1.10906 \times 10^{-13}$ & $1.54844 \times 10^{-13}$ & $3.59431 \times 10^{-14}$ \\
$(0.3,0.3)$ & $8.16245 \times 10^{-13}$ & $8.19524 \times 10^{-13}$ & $7.92034 \times 10^{-13}$ & $3.47283 \times 10^{-13}$ \\
$(0.4,0.4)$ & $5.3667 \times 10^{-13}$ & $6.55282 \times 10^{-13}$ & $6.19716 \times 10^{-13}$ & $2.84722 \times 10^{-13}$ \\
$(0.5,0.5)$ & $1.21825 \times 10^{-12}$ & $1.27512 \times 10^{-12}$ & $1.23951 \times 10^{-12}$ & $7.10487 \times 10^{-13}$ \\
$(0.6,0.6)$ & $6.22051 \times 10^{-13}$ & $7.56301 \times 10^{-13}$ & $6.9298 \times 10^{-13}$ & $3.39517 \times 10^{-13}$ \\
$(0.7,0.7)$ & $1.20544 \times 10^{-14}$ & $1.32987 \times 10^{-12}$ & $1.22743 \times 10^{-12}$ & $6.05743 \times 10^{-13}$ \\
$(0.8,0.8)$ & $1.13173 \times 10^{-13}$ & $1.24659 \times 10^{-12}$ & $1.1751 \times 10^{-12}$ & $5.70221 \times 10^{-13}$ \\
$(0.9,0.9)$ & $1.34524 \times 10^{-12}$ & $1.48586 \times 10^{-12}$ & $1.40536 \times 10^{-12}$ & $7.08918 \times 10^{-13}$ \\
\hline
\end{tabular}

\section{Table 1}

Residual error values $E(x, t)$ for the solution of the initial-boundary value problem (2)-(3) at a collection of points $(x, t)$ in the problem domain. We have taken the initial condition to be $u(x, 0)=f(x)=\sin (\pi x)$ and have considered various values of the parameter $\lambda$. 


\begin{tabular}{cccc}
\hline & $E(x, t)$ & $E(x, t)$ & $E(x, t)$ \\
$(x, t)$ & for $\lambda=0.3$ & for $\lambda=1.0$ & for $\lambda=8.0$ \\
\hline$(0.1,0.1)$ & $1.21098 \times 10^{-11}$ & $1.39757 \times 10^{-11}$ & $9.31578 \times 10^{-12}$ \\
$(0.2,0.2)$ & $1.7906 \times 10^{-11}$ & $2.15371 \times 10^{-11}$ & $2.30782 \times 10^{-11}$ \\
$(0.3,0.3)$ & $4.60745 \times 10^{-11}$ & $5.14205 \times 10^{-11}$ & $1.14156 \times 10^{-12}$ \\
$(0.4,0.4)$ & $6.31725 \times 10^{-11}$ & $6.99688 \times 10^{-11}$ & $1.14303 \times 10^{-11}$ \\
$(0.5,0.5)$ & $7.38272 \times 10^{-11}$ & $8.18388 \times 10^{-11}$ & $1.62139 \times 10^{-11}$ \\
$(0.6,0.6)$ & $7.6835 \times 10^{-11}$ & $8.56434 \times 10^{-11}$ & $2.74148 \times 10^{-11}$ \\
$(0.7,0.7)$ & $8.60242 \times 10^{-11}$ & $9.58326 \times 10^{-11}$ & $3.12081 \times 10^{-11}$ \\
$(0.8,0.8)$ & $1.09004 \times 10^{-12}$ & $1.20004 \times 10^{-10}$ & $2.17772 \times 10^{-11}$ \\
$(0.9,0.9)$ & $1.14691 \times 10^{-11}$ & $1.26796 \times 10^{-11}$ & $2.93794 \times 10^{-11}$ \\
\hline
\end{tabular}

Table 2

Residual error values $E(x, t)$ for the solution of the initial-boundary value problem (2)-(3) at a collection of points $(x, t)$ in the problem domain. We have taken the initial condition to be $u(x, 0)=f(x)=x^{3}(1-x)^{3}$ and have considered various values of the parameter $\lambda$. 


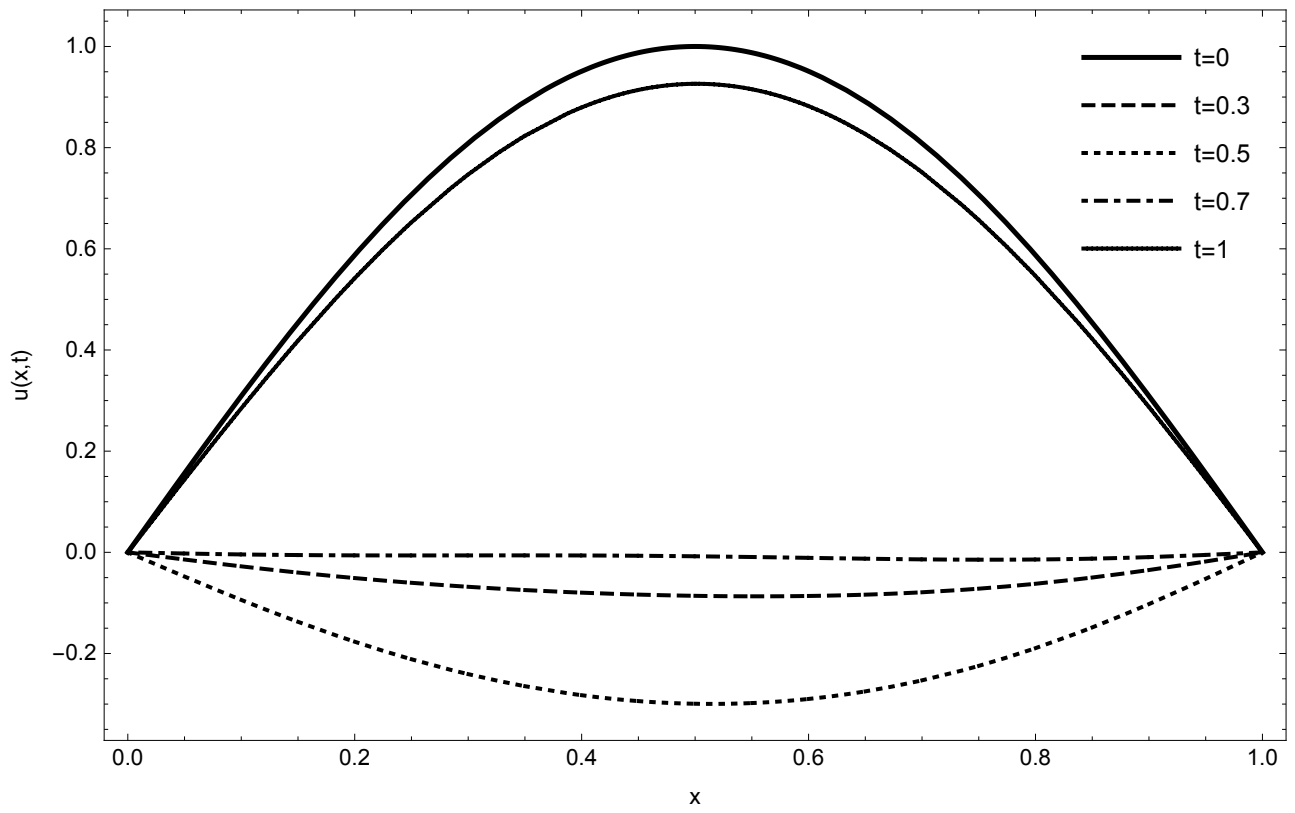

Fig. 1. Plot of a 20-term reproducing kernel method approximation to the initial-boundary value problem (2)-(3). We have taken the initial condition to be $u(x, 0)=f(x)=\sin (\pi x)$, while the parameter is chosen as $\lambda=0.3$.

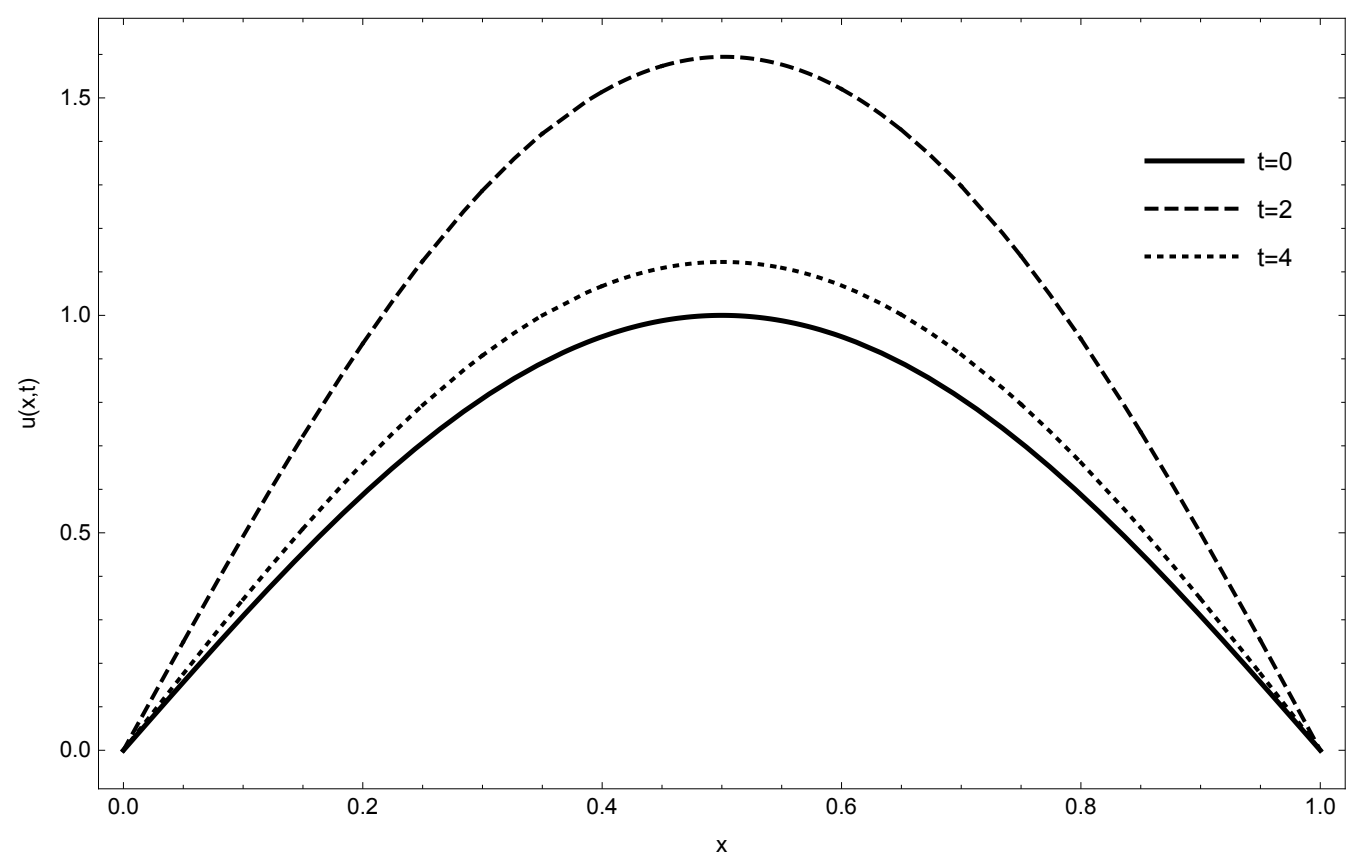

Fig. 2. Plot of a 20-term reproducing kernel method approximation to the initial-boundary value problem (2)-(3). We have taken the initial condition to be $u(x, 0)=f(x)=\sin (\pi x)$, while the parameter is chosen as $\lambda=1$. 


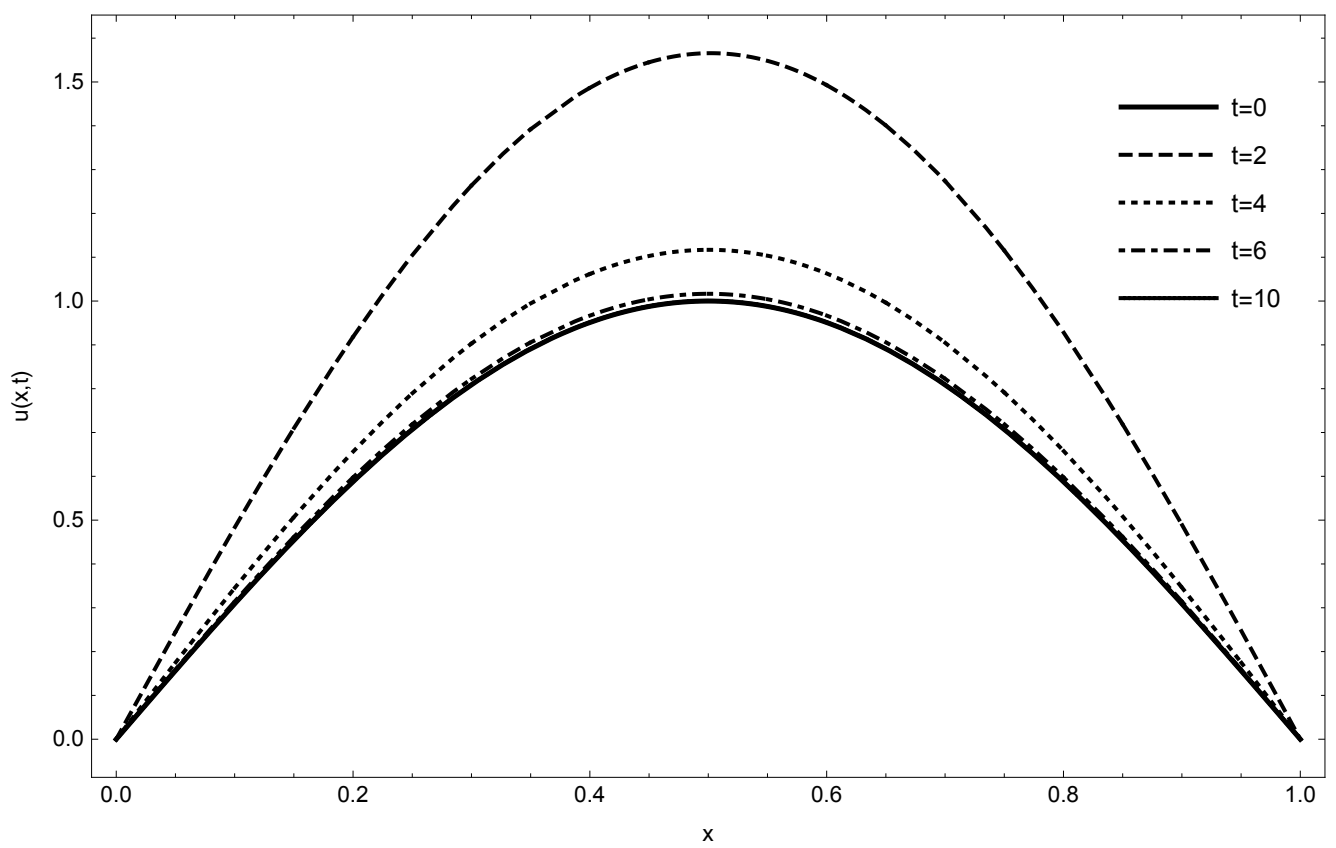

Fig. 3. Plot of a 20-term reproducing kernel method approximation to the initial-boundary value problem (2)-(3). We have taken the initial condition to be $u(x, 0)=f(x)=\sin (\pi x)$, while the parameter is chosen as $\lambda=8$.

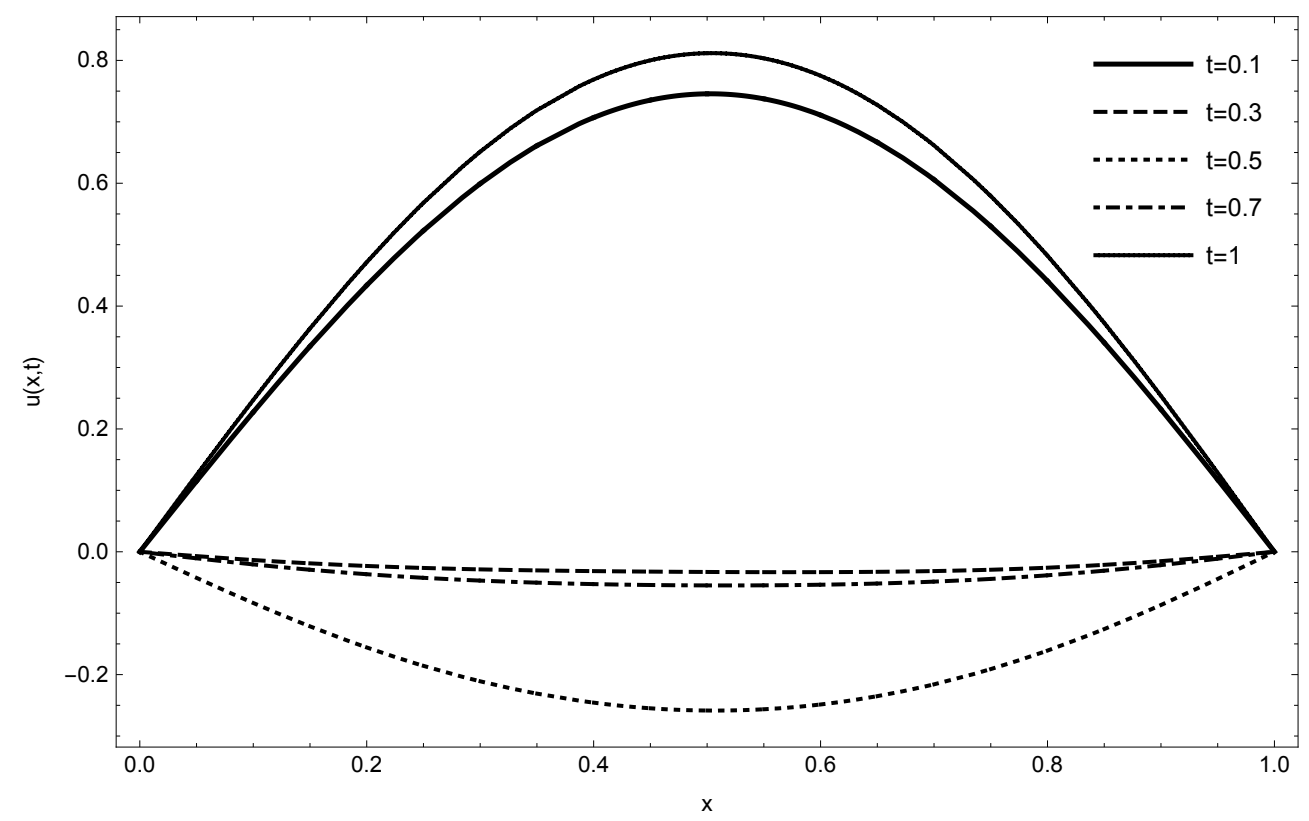

Fig. 4. Plot of a 20-term reproducing kernel method approximation to the initial-boundary value problem (2)-(3). We have taken the initial condition to be $u(x, 0)=f(x)=x^{3}(1-x)^{3}$, while the parameter is chosen as $\lambda=0.3$. 


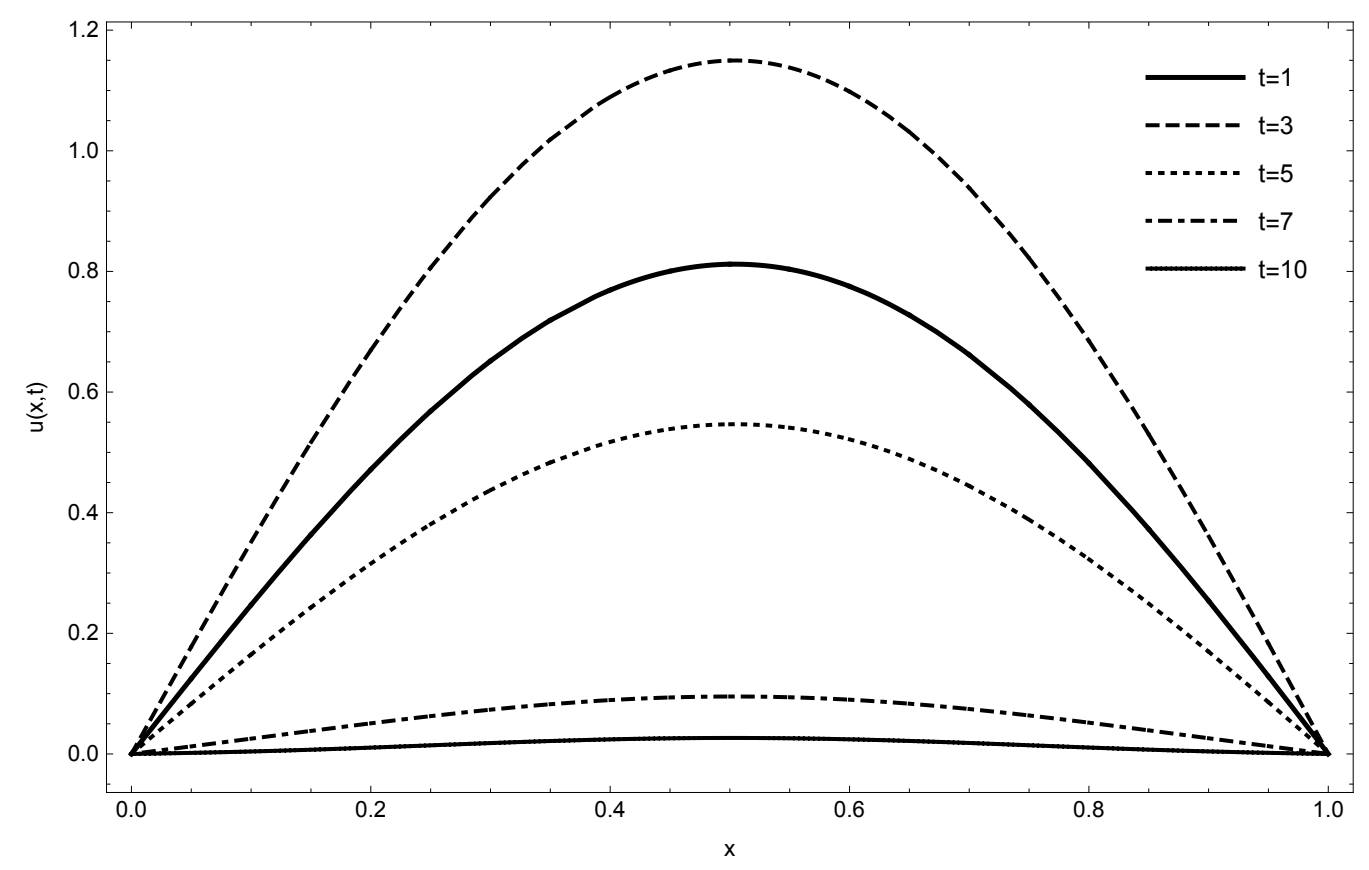

Fig. 5. Plot of a 20-term reproducing kernel method approximation to the initial-boundary value problem (2)-(3). We have taken the initial condition to be $u(x, 0)=f(x)=x^{3}(1-x)^{3}$, while the parameter is chosen as $\lambda=1$.

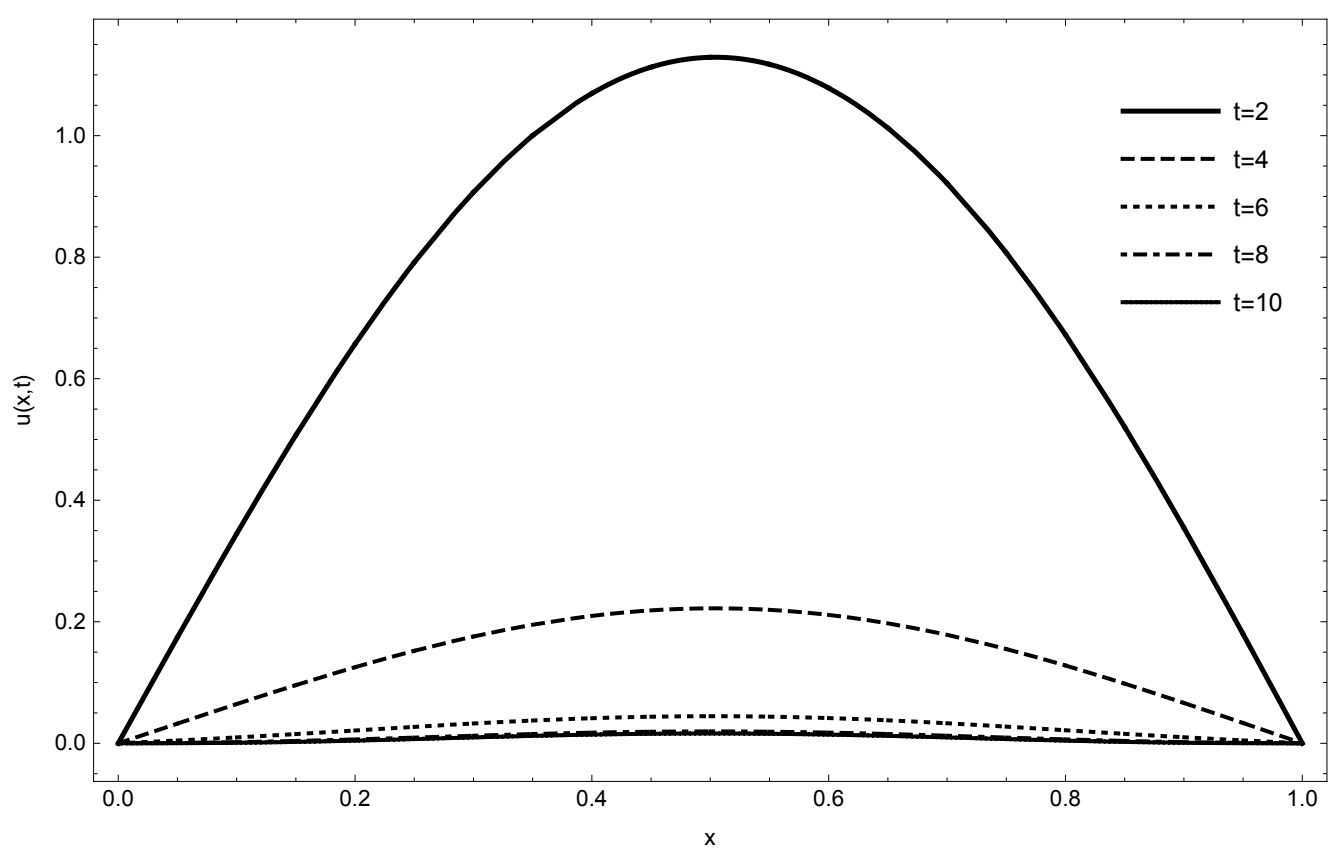

Fig. 6. Plot of a 20-term reproducing kernel method approximation to the initial-boundary value problem (2)-(3). We have taken the initial condition to be $u(x, 0)=f(x)=x^{3}(1-x)^{3}$, while the parameter is chosen as $\lambda=8$. 